Животноводство и кормопроизводство 2021 / Animal Husbandry and Fodder Production 2021;104(4) 108 ТЕОРИЯ И ПРАКТИКА КОРМЛЕНИЯ/ТНЕОRY AND PRACTICE ОF FEЕDING

Животноводство и кормопроизводство. 2021. Т. 104, № 4. С. 108-123.

Animal Husbandry and Fodder Production. 2021. Vol. 104, no 4. P. 108-123.

Обзорная статья

ТЕОРИЯ И ПРАКТИКА КОРМЛЕНИЯ

УДК 636.085:612.017.11/.12

doi: $10.33284 / 2658-3135-104-4-108$

Фитобиотики как альтернатива антибиотикам в животноводстве

Виталий Александрович Рязанов ${ }^{1}$, Марина Яковлевна Курилкина ${ }^{2}$, Галимжан Калиханович Дускаев ${ }^{3}$, Вячеслав Михайлович Габидулин

1,23,4, Федеральный научный центр биологических систем и агротехнологий Российской академии наук, Оренбург, Россия ${ }_{1}^{1}$ vita7456@yandex.ru, https://orcid.org/0000-0003-0903-9561

${ }^{2} \mathrm{~K}$ marina4@mail.ru,https://orcid.org/0000-0003-0253-7867

3gduskaev@mail.ru, https://orcid.org/0000-0002-9015-8367

4Gabidulin.V.M@yandex.ru https://orcid.org/0000-0003-0284-1084

Аннотаиия. На протяжении последних десятилетий основным способом предотврашения болезней с целью улучшения производственных показателей в животноводстве является использование антибиотиков. Между тем из-за необоснованного использования последних сушествует риск vвеличения числа устойчивых к антибиотикам бактерий. В этой связи практически повсеместно. последовательно реализуется тенденция к полному или частичному отказу от таких препаратов. В последние годы многие исследователи. работаюшие в области кормления сельскохозяйственных животных, обратили внимание на биологически активные вешества, образуюшиеся в растениях фитобиотики. Доказано. что фитобиотики улучшают функшии иммунной системы. оказывают значительное влияние на состояние здоровья животных и повышают продуктивные качества. К числу факторов, определяющих актуальность разработки высокоэффективных фитобиотиков в животноводстве, можно отнести то, что глобальное использование фитобиотиков в качестве альтернативы различным противомикробным препаратам приведёт не только к защите здоровья и получению максимальной продуктивности животных, но также обеспечит решение задач общественного здравоохранения, включая проблему повышения безопасности пищевых продуктов, за счёт исключения кумулятивных эффектов от антибиотиков в продуктах животного происхождения (молоко, мясо). В данном обзоре рассматривается возможность использования фитобиотических добавок в качестве альтернативы обычным противомикробным препаратам (антибиотикам), в том числе при возникновении бактериальных инфекций со множественной лекарственной устойчивостью.

Ключевые слова: животноводство, кормление животных, фитобиотики, растительные экстракты, кормовые антибиотики, резистентность, продуктивность

Благодарности: работа выполнена при поддержке Российского научного фонда, проект № 21-76-10014.

Для цитирования: Рязанов В.А., Курилкина М.Я., Дускаев Г.К., Габидулин В.М. Фитобиотики как альтернатива антибиотикам в животноводстве (обзор) // Животноводство и кормопроизводство. 2021. Т. 104, № 4. С. 108-123. https://doi.org/10.33284/2658-3135-104-4-108

Original artice

\title{
THEORY AND PRACTICE OF FEEDIND
}

Phytobiotics as an alternative to antibiotics in animal husbandry (review)

Vitaly A Ryazanov ${ }^{1}$, Marina Ya Kurilkina ${ }^{2}$, Galimzhan K Duskaev ${ }^{3}$, Vyacheslav M Gabidulin ${ }^{4}$

${ }_{1,2,3,4}$ Federal Research Centre ofBiological Systems and Agrotechnologies of the Russian Academy of Sciences, Orenburg, Russia

${ }^{1}$ vita7456@yandex.ru, https://orcid.org/0000-0003-0903-9561

${ }^{2}$ K_marina4@mail.ru, https://orcid.org/0000-0003-0253-7867

3.gduskaev@mail.ru, https://orcid.org/0000-0002-9015-8367

${ }^{4}$ Gabidulin.V.M@yandex.ru https://orcid.org/0000-0003-0284-1084

Abstract. Over the past decades, the use of antibiotics has been the main way to prevent disease in order to improve performance in livestock production. However, due to the unjustified use of the latter,

СРязанов В.А., Курилкина М.Я., Дускаев Г.К., Габидулин В.М., 2021 
there is a risk to increase the number of antibiotic-resistant bacteria. In this regard, almost everywhere, the tendency towards complete or partial rejection of such drugs is consistently implemented. In recent years, many researchers working in the field of feeding of farm animals have paid attention to biologically active substances formed in plants - phytobiotics. It has been proven that phytobiotics improve the functions of the immune system, have a significant effect on the health of animals and increase productive qualities. Among the factors that determine the relevance of the development of highly effective phytobiotics in animal husbandry, it is possible to attribute the fact that the global use of phytobiotics as an alternative to various antimicrobial drugs will not only lead to the protection of health and maximize animal productivity, but will also ensure the solution of public health problems, including the problem of improving safety. food products, by eliminating the cumulative effects of antibiotics in animal products (milk, meat). This review examines the possibility of using phytobiotic supplements as an alternative to conventional antimicrobial drugs (antibiotics), including in the case of multidrug-resistant bacterial infections.

Keywords: animal husbandry, animal feeding, phytobiotics, plant extracts, feed antibiotics, resistance, productivity

Acknowledgments: the work was supported by the Russian Science Foundation, Project № 21-76-10014.

For citation: Ryazanov VA, Kurilkina MYa, Duskaev GK, Gabidulin VM. Phytobiotics as an alternative to antibiotics in animal husbandry (review). Animal Husbandry and Fodder Production. 2021;104(4):108-123. (In Russ.). https://doi.org/10.33284/2658-3135-104-4-108

\section{Введение.}

С момента открытия и применения пенициллина антибиотики сыграли беспрецедентную роль в профилактике, контроле и лечении инфекционных заболеваний. Доказано, что использование антибиотиков в кормлении животных является эффективным методом повышения усвояемости кормов, стимулирования роста животных и улучшения качества получаемой продукции (Lin J, 2014). Однако необоснованное использование антибиотиков вызывает опасения в части развития резистентных бактерий, которые могут привести к передаче резистентных факторов от животных к человеку (Stanton TB, 2013). Не терапевтическое использование противомикробных препаратов также связано с распространением множественной лекарственной устойчивости, включая устойчивость к лекарствам, которые никогда не использовались в животноводстве (Marshall BM and Levy SB, 2011). По этой причине Швеция впервые запретила использование некоторых антибиотиков в кормах для животных в 1986 году (Castanon JI, 2007), а страны-члены Европейского союза (EC) запретили использование всех стимуляторов роста, антибиотиков в 2006 году в соответствии с Постановлением Европейского парламента и Совета ЕС № 1831/2003. Между тем, общее количество применяемых антибиотиков в животноводстве увеличилось, поскольку в результате запрета возникла высокая частота заболеваний сельскохозяйственных животных. В отличие от так называемого «золотого века антибиотиков», когда были открыты и коммерциализированы многие классы антибиотиков, разработка и выпуск новых антибиотиков за последние десятилетия резко сократилась (Borchardt RA and Rolston KV, 2013). Например, более 40 лет не было новых классов антибиотиков для подавления грамотрицательных бактерий, таких как Escherichia coli и Salmonella enterica. В то же время в медицинских и ветеринарных клиниках были выявлены штаммы этих бактерий с широкой лекарственной устойчивостью и резистентностью (устойчивые ко всем терапевтическим антибиотикам) (Cooper MA and Shlaes D, 2011).

Чтобы преодолеть повышенный уровень смертности и заболеваемости животных из-за запрета на добавление антибиотиков в корма сельскохозяйственным животным, возросла необходимость в создании возможных альтернатив антибиотикам (Seal BS et al., 2013; Дускаев Г.К. и др., 2019). В последние годы многие исследователи, работающие в области кормления сельскохозяйственных животных, обратили внимание на биологически активные вещества, образующиеся в растениях - фитобиотики (Abreu AC et al., 2012; Cheng G et al., 2014). Доказано, что фитобиотики улучшают функции иммунной системы, оказывают значительное влияние на состояние здоровья животных и повышают продуктивные качества (Windisch W et al., 2008; Stevanović ZD et al., 2018). К числу факторов, определяющих актуальность разработки высокоэффективных фитобиотиков в 
Животноводство и кормопроизводство 2021 / Animal Husbandry and Fodder Production 2021;104(4) 110 ТЕОРИЯ И ПРАКТИКА КОРМЛЕНИЯ/ТНЕОRY AND PRAСТICЕ ОF FЕEDING

животноводстве, можно отнести то, что глобальное использование фитобиотиков в качестве альтернативы различным противомикробным препаратам приведёт не только к защите здоровья и получению максимальной продуктивности животных, но также обеспечит решение задач общественного здравоохранения, включая проблему повышения безопасности пищевых продуктов, за счёт исключения кумулятивных эффектов от антибиотиков в продуктах животного происхождения (молоко, мясо) (Hashemi SR and Davoodi H, 2011; Kiczorowska B et al., 2016; Багно О.А. и др., 2018).

В данном обзоре рассматривается мировой опыт использования в животноводстве фитобиотических добавок в качестве альтернативы обычным противомикробным препаратам (антибиотикам).

В последние годы использование экстрактов лекарственных растений в животноводстве увеличилось вследствие выявления новых побочных эффектов современных антибиотиков, связанных с аккумуляцией их токсичных остатков в продуктах питания, а также по причине повышения антибиотикорезистентности микроорганизмов (González JA et al., 2011; Kuralkar P and Kuralkar SV, 2021; Duskaev GK et al., 2021). Фитобиотики обладают полезными, многофункциональными свойствами, которые в первую очередь связаны с наличием в их составе различных биологически активных метаболитов или фитосоединений, а именно гликозиды и алкалоиды (спирты, альдегиды, сложные эфиры, простые эфиры, кетоны, лактоны и т. д.), антоцианы, кумарины, флавоноиды, фенольные соединения (дубильные вещества, танины), сапонины и терпеноиды (моно- и сесквитерпены, стероиды и т. д.) (Dhama K et al., 2014; Singh J and Gaikwad DS, 2020).

Общей чертой фитобиотиков является то, что они представляют собой очень сложную смесь биологически активных компонентов. Состав фитобиотиков может варьироваться в зависимости от биологических факторов (виды растений, место произрастания и условия сбора урожая), производства (экстракция/дистилляция и стабилизация) и условий хранения (свет, температура, давление и время) и только при определённых обстоятельствах экстракты растений могут улучшить продуктивность животных и контролировать болезни (Huyghebaert G et al., 2011; Cross DE et al., 2007). Как показал настоящий анализ литературных источников, эффективность применения фитобиотиков в животноводстве в основном зависит от используемой части растений (стебель, лист, корень), генетической вариации, возраста, используемой дозировки, метода экстракции, время сбора урожая и совместимости с другими ингредиентами (Yang Y et al., 2009). Кроме того, на положительный эффект растительных фитобиотиков может влиять состав рациона, условия окружающей среды, а также наличие тех или иных инфекционных заболеваний (Giannenas AI et al., 2003; Sarica S et al., 2005).

Литературные данные свидетельствуют, что травяные препараты могут действовать не только как пищевые добавки, но и как средства предотвращения или контроля различных бактериальных инфекций (особенно кишечных и респираторных), инфекций, вызванных отдельными кишечными патогенами, в частности E. Coli, S. aureus, E. Aerogenes, P. aeruginosa, B. Subtilis, K. pneumoniae, S. typhi, S. epidermidis, S. Typhimurium и P. Vulgaris. Было установлено, что практически все изученные экстракты растений (водно-спиртовые экстракты лекарственных растений), а именно: Achyranthes aspera (A. aspera) L. (семейство Asparagaceae), Ficus caria (F. caria) (семейство Moraceae), Malvi parviflora (M. parviflora) (семейство Malvaceae), видов Vernonia (местное название Alakit, семейство Asteraceae), Calpurinia aurea (C. aurea) (Ait) Benth (семейство Fabaceae), Nicotiana tabacum (N. tabacum) L. (семейство Solanaceae), Ziziphus spina-christi (Z. spina-christi) (ceмейство Rhamnaceae), Croton macrostachys (C. macrostachys) (семейство Euphorbiaceae), за исключением S. hastifolium, проявляют аналогичный антибактериальный эффект. Вместе с тем, 30 \% отобранных экстрактов трав продемонстрировали антибактериальную активность в условиях in vitro против MDR-A. Baumannii (Miyasaki Y et al., 2010). Также сообщалось, что фитосоединения проявляют сильную антибактериальную активность против метициллинрезистентных бактерий (МРЗС) (Kipre BG et al., 2017) и бактерий, продуцирующих бета-лактамазы расширенного спектра действия (Upadhyaya I et al., 2015). 
Побочные продукты растительной пищи также используются в качестве объекта для регуляции бактериального статуса организма животных (Guil-Guerrero JL et al., 2016a; 2016b). В частности, экстракты побочных продуктов граната действуют как хороший источник фенольных соединений, обладающих антиоксидантной, противовоспалительной и антимикробной активностью (Reddy MK et al., 2007). В прошлом несколько фитокомпонентов показали многообещающую антибактериальную активность против штаммов с множественной лекарственной устойчивостью.

По этой причине многие из протестированных растений широко используются в ветеринарии для лечения заболеваний различной этиологии (Kalayou S et al., 2012; Rahal A et al., 2014). B частности, было показано, что масло чайного дерева и его активный компонент, терпинен-4-ол, повышали эффективность лечения мастита крупного рогатого скота за счёт ингибирования провоспалительных цитокинов, а также усиления экспрессии противовоспалительных цитокинов (Таga I et al., 2012). В более раннем исследовании аллицин был эффективно применён при лечении диареи новорождённых телят, вызванной Cryptosporidium parvum (Ghosh S et al., 2010; 2011). Аналогичный эффект был получен от применения луковиц Elephantor rhizaburkei и коры Xanthocersis zambesiaca (Panda SS and Dhal NK, 2014; O'Bryan CA et al., 2015). Масштабные исследования по оценке эффективности применения экстрактов десяти растений, используемых для предотвращения и контроля бактериальных маститов крупного рогатого скота в Южной Бразилии, показали, что экстракты Alternanthera brasiliana, Achillea millefolium, Baccharis trimera и Solidago chilensis были активными против S. aureus, тогда как Symphythum officinale, Sambucus nigra, Mentha sp., Ocimum basilicum, Parapiptadenia rigida и Cuphea carthagenensis были активны против обоих микроорганизмов (Papatsiros VG et al., 2013). В других исследованиях были продемонстрированы антиоксидантные, противовоспалительные, гипогликемические, противоопухолевые и противомикробные свойства диферулоилметана, полифенола, выделенного из корневищ куркумы (Curcuma longa) (Ghosh S et al., 2015).

Luseba D and Tshisikhawe MP (2013) проанализировав имеющиеся данные о лекарственных растениях, используемых для лечения заболеваний крупного рогатого скота в Южной Африке, установили, что экстракты листьев и корней Asparagus falcatus, Tagetes minuta, Diospyro slycioides и Vernonia corymbosa могут применяться для борьбы с клещами и гельминтами у крупного рогатого скота. При этом, несмотря на широкий перечень заболеваний, вылечиваемых посредством фитобиотиков, до настоящего времени лишь в отдельных исследованиях отражена эффективность их применения для лечения особо опасных инфекций, таких как сибирская язва, эфемерная лихорадка, ящур т. д. (Panda SS and Dhal NK, 2014).

Помимо других растительных компонентов, широко распространённой практикой становится использование растительных эфирных масел для повышения эффективности использования корма жвачными животными. Эфирные масла давно известны своими антимикробными свойствами (Lee KW et al., 2004), но точный механизм действия противомикробных препаратов ещё недостаточно изучен (Stavri M et al., 2007). Некоторые наблюдения in vivo подтверждают предположение, что общий антимикробный потенциал эфирных масел способствует окончательному снижению давления кишечных патогенов (Windisch W et al., 2008). К числу основных функциональных мишеней для эфирных масел в организме крупного рогатого скота принято относить регуляцию пищеварения за счёт увеличения высвобождения пищеварительных ферментов, доступных для роста бактерий в просвете кишечника. В частности, Nanon A с коллегами (2014) документально подтвердили, что эфирные масла из лимонной травы и смеси чеснока и имбиря могут использоваться в качестве модификатора рубца, что приводит к улучшению переваривания грубых кормов (Sethiya NK et al., 2016; Diniz do Nascimento et al., 2020).

Биоактивные растительные вторичные соединения добавок, таких как алкалоиды, флавоноиды, сапонины, дубильные вещества, фенолы, терпеноиды, эфирные масла и пиперин улучшают метаболизм белков и снижают выработку метана, уменьшают пищевой стресс, что в конечном итоге оптимизирует ферментацию рубца, улучшая здоровье и продуктивность животных (Kumar et al., 2013). Данный факт лёг в основу их успешного применения в составе кормовых добавок для по- 
Животноводство и кормопроизводство 2021 / Animal Husbandry and Fodder Production 2021;104(4) 112 ТЕОРИЯ И ПРАКТИКА КОРМЛЕНИЯ/ТНЕОRY AND PRAСТICЕ ОF FЕEDING

вышения метаболизма и эффективности использования кормов жвачными животными (Атландерова К.Н. и др. 2019; Singh PK et al., 2020; Ryazanov VA et al., 2020; 2021). В частности, было показано, что скармливание молодняку крупного рогатого скота экстракта Quercus cortex и комплекса веществ ингибиторов Quorum sensing благоприятно влияет на скорость обмена азота и стимулирует нарастание содержания аммиака в рубце подопытных животных, что сопровождается повышением переваримости сухого вещества, протеина и клетчатки (Atlanderova KN et al., 2019b). Однако, несмотря на всю перспективность данного направления, есть свидетельства того, что некоторые эфирные масла снижают скорость дезаминирования аминокислот, а также скорость производства аммиака путём подавления популяций бактерий, продуцирующих аммиак.

У животных с однокамерным желудком фитобиотические кормовые добавки снижают патогенную нагрузку в кишечнике, стимулируя образование полезных кишечных микробов и, как следствие, увеличивают переваримость кормов (Lange et al., 2010). Некоторые из авторов сообщили о положительном влиянии фитохимических соединений на усвояемость питательных веществ за счёт увеличения переваримости сырого протеина (Mourao JL et al., 2006; Maenner K et al., 2011; Emami N K et al., 2012; Li P et al., 2012). У таких сельскохозяйственных животных, как свиньи и птица, эфирные масла усиливают выработку пищеварительных секретов и повышают усвоение питательных веществ, снижая патогенный стресс в кишечнике (Zeng Z et al., 2015). Также показано, что экстракты куркумы, имбиря и чеснока положительно влияют на продуктивность, микробную нагрузку и морфологию кишечника свиней-отъемышей (Yan L et al., 2011; Adebiyi OA et al., 2014). Позднее в экспериментах in vitro установлено, что при введение экстракта коры дуба (Quercus cortex) в разных дозировках в руминальную жидкость, наблюдается повышение усвояемости кормов с 2,42 до $7,13 \%$, при этом отмечается значительное повышение показателя $\mathrm{pH}$ в руминальной жидкости до значения 6,8 единиц, что в конечном итоге способствует увеличению усвояемости питательных веществ (Atlanderova KN et al., 2019a; 2021). Однако большинство имеющихся результатов исследований in vitro требует дальнейших исследований на модели in vivo для проверки и подтверждения имеющихся данных.

В последние годы фитобиотические препараты всё чаще рассматриваются в качестве природных стимуляторов роста и продуктивных качеств сельскохозяйственных животных и могут стать многообещающей заменой антибиотических стимуляторов роста в современном животноводстве. В частности, недавнее исследование, проведённое Jeshari M с коллегами (2016), показало, что добавление к стартовому рациону телят голштинской породы смеси эфирных масел из Rosmarinus officinalis L., Zataria multiflora Boiss и Mentha pulegium L. положительно влияет на показатели роста подсосных телят. Результаты исследований Тарki I с соавторами (2020) показали, что эфирное масло орегано является эффективной жидкой кормовой добавкой, улучшающей состояние здоровья и показатели роста телят голштино-фризской породы.

Введение в рацион свиней корицы, мексиканского перца, тимьяна и карвакрола уменьшает массу патогенных микробов в кишечнике; сангровит, экстракт выдержанного чеснока и аллицина способны увеличивать набор массы тела животных (Namkung H et al., 2004; Manzanilla EG et al., 2004; Oetting LL et al., 2006; Costa LB et al., 2007). Также сообщается о влиянии фитогенных кормовых добавок на продуктивность сельскохозяйственной птицы (Hashemi SR and Davoodi H, 2010). Так, Singh PK с коллегами (2019) установили, что диетические добавки из чёрного тмина, чеснока и куркумы не только влияют на яйценоскость и качество яиц, но также помогают снизить уровень холестерина и триглицеридов яичного желтка в течение первой фазы периода яйцекладки. Травяные кормовые добавки (чёрный тмин, чеснок и куркума) в сочетании с льняным маслом положительно влияют на яйценоскость, потребление корма и массу яйца (Ghosh T et al., 2020). Al-Kassie GAM (2010) установил, что включение сухой мяты перечной (Mentha piperita L.) в рацион цыплятбройлеров улучшает прирост живой массы и увеличивает коэффициент конверсии корма. Добавление в рацион бройлеров гудучи (Tinospora cordifolia) положительно влияет на весовой рост, показатели качества и сроки хранения мяса (Saeed M et al., 2020). 
В целом, на сегодняшний день во всём мире наблюдается значительный рост использования растительных экстрактов. Крупный рогатый скот, лошади, овцы, козы и свиньи составляют около $70 \%$ животных, получающих лечебные травы, за ними следуют домашняя птица $-9,1 \%$, собаки $-5,3$ \% и кролики - 4,3 \% (Viegi L et al., 2003). Это связано не только с общей тенденцией к использованию натуральных продуктов для лечения болезней, но и с наличием значительных доказательств эффективности лечебных трав. Таким образом, использование растительных экстрактов становится всё более актуальным вопросом в области животноводства (Laudato M and Capasso R, 2013; Kumar M et al., 2014).

\section{Заключение.}

1. Всестороннее изучение свойств растений, содержащих фитобиотические компоненты, использование современных технологий для получения и стандартизации этих компонентов, их экспериментальная и производственная апробация позволяют широко применять растительные экстракты в кормлении животных в качестве биологически активных добавок последнего поколения на основе сырья естественного происхождения.

2. В условиях интенсивных технологий животноводства фитобиотики нивелируют такие явления, как снижение иммунного и антиоксидантного статуса животных, обеспечивают повышение всех видов продуктивности за счёт улучшения потребления, переваримости, усвояемости кормов, нормализации кишечной микрофлоры и гомеостаза в целом.

\section{Список источников}

1. Использование пробиотиков и растительных экстрактов для улучшения продуктивности жвачных животных (обзор) / Г.К. Дускаев, Г.И. Левахин, В.Л. Королёв, Ф.Х. Сиразетдинов // Животноводство и кормопроизводство. 2019. Т. 102. № 1. С. 136-148. [Duskaev GK, Levakhin GI, Korolyov VL, Sirazetdinov FKh. Use of probiotics and plant extracts to improve the productivity of ruminants (review). Animal Husbandry and Fodder Production. 2019;102(1):136-148. (In Russ)]. doi: $10.33284 / 2658-3135-102-1-136$

2. Микробиом рубца крупного рогатого скота при использовании в кормлении экстракта Quercus cortex / К.Н. Атландерова, Г.К. Дускаев, А.М. Макаева, Д.М. Муслюмова, К.С. Кондрашова // Животноводство и кормопроизводство. 2019. Т. 102. № 4. С. 186-197. [Atlanderova KN, Duskaev GK, Makaeva AM, Muslyumova DM, Kondrashova KS. Cattle rumen microbiome after Quercus cortex extract. Animal Husbandry and Fodder Production. 2019;102(4):186-197. (In Russ)]. doi: 10.33284 / 2658-3135-102-4-186

3. Фитобиотики в кормлении сельскохозяйственных животных (обзор) / О.А. Багно, О.Н. Прохоров, С.А. Шевченко, А.И. Шевченко, Т.В. Дядичкина // Сельскохозяйственная биология. 2018. Т. 53. № 4. С. 687-697. doi: 10.15389/agrobiology.2018.4.687rus [Bagno OA, Prokhorov ON, Shevchenko SA, Shevchenko AI, Dyadichkina TV. Use of phytobioticts in farm animal feeding (review). Sel'skokhozyaistvennaya biologiya [Agricultural Biology]. 2018;53(4):687-697. (In Russ)]. doi: 10.15389/agrobiology.2018.4.687eng

4. Abreu AC, McBain AJ, Simoes M. Plants as sources of new antimicrobials and resistancemodifying agents. Nat Prod Rep. 2012(9);29:1007-1021. doi: 10.1039/c2np20035j

5. Adebiyi OA, Ajayi OS, Adejumo IO, Osungade TO. Performance, microbial load and gut morphology of weaned pigs fed diets supplemented with turmeric, ginger and garlic extract. Trop Anim Prod. Investig. 2014;17(1):25-31.

6. Al-Kassie GAM. The role of peppermint (Mentha piperita) on performance in broiler diets. Agric Biol J N Am. 2010;1(5):1009-1013.

7. Atlanderova KN, Makaeva AM, Kurilkina MYa, Kholodilina TN, Duskaev GK. Technology for increasing the bioavailability of feed using quorum sensing inhibitors. IOP Conference Series: Earth and Environmental Science: International Conference on World Technological Trends in Agribusiness 
Животноводство и кормопроизводство 2021 / Animal Husbandry and Fodder Production 2021;104(4) 114 ТЕОРИЯ И ПРАКТИКА КОРМЛЕНИЯ/ТНЕОRY AND PRAСТICЕ ОF FЕЕDING

4-5 July 2020, Omsk City, Western Siberia, Russian Federation. Bristol, England: IOP Publishing; 2021;624:012159. doi: 10.1088/1755-1315/624/1/012159

8. Atlanderova KN, Makaeva AM, Sizova EA, Duskaev GK. Stimulation of ruminal digestion of young cattle with oak bark extract (Quercus cortex). IOP Conference Series: Earth and Environmental Science: Conference on Innovations in Agricultural and Rural development; 2019 Apr 18-19; Kurgan, Russian Federation. Bristol, England: IOP Publishing; 2019a;341:012059. doi: 10.1088/1755-1315/341/1/012059

9. Atlanderova KN, Makaeva AM, Sizova EA. Comparative evaluation of the effect of the Quercus cortex extract and biologically active substances of plant origin on health and scar digestion. IOP Conference Series: Earth and Environmental Science: Conference on Innovations in Agricultural and Rural development; 2019 Apr 18-19; Kurgan, Russian Federation. Bristol, England: IOP Publishing; 2019b;341:012189. doi: 10.1088/1755-1315/341/1/012189

10. Borchardt RA, Rolston KV. Antibiotic shortages: effective alternatives in the face of a growing problem. Jaapa. 2013;26(2):13-18. doi: 10.1097/01720610-201302000-00004

11. Castanon JI. History of the use of antibiotic as growth promoters in European poultry feeds. Poult. Sci. 2007;86(11):2466-2471. doi: 10.3382/ps.2007-00249

12. Cheng G, Hao H, Xie S, Wang X, Dai M, Huang L, Yuan Z. Antibiotic alternatives: the substitution of antibiotics in animal husbandry? Front Microbiol. 2014;5:69-83. doi: 10.3389/fmicb.2014.00217

13. Cooper MA, Shlaes D. Fix the antibiotics pipeline. Nature. 2011;472:32. doi: $10.1038 / 472032 \mathrm{a}$

14. Costa LB, Panhoza Tse ML, Miyada VS. Herbal extracts as alternatives to antimicrobial growth for weanling pigs. Braz J Anim Sci. 2007;36(3):589-595. doi: 10.1590/S1516-35982007000300011

15. Cross DE, Mcdevitt RM, Hillman K, Acamovic T. The effect of herbs and their associated essential oils on performance, dietary digestibility and gut microflora in chickens from 7 to 28 days of age. Br Poult Sci. 2007;48(4):496-506. doi: 10.1080/00071660701463221

16. Dhama K, Tiwari R, Chakraborty S, Saminathan M, Kumar A, Karthik K, Yaqoob M, Amarpal W, Singh SV, Rahal A. Evidence based antibacterial potentials of medicinal plants and herbs countering bacterial pathogens especially in the era of emerging drug resistance: an integrated update. Int $\mathrm{J}$ pharmacology. 2014;10(1):1-43. doi: 10.3923/ijp.2014.1.43

17. Diniz do Nascimento L, Moraes A, Costa K, Pereira JM, Galúcio, Taube PS, Costa C, Neves Cruz J, de Aguiar Andrade EH, Faria L. Bioactive natural compounds and antioxidant activity of essential oils from spice plants: new findings and potential applications Biomolecules. 2020;10(7):988. doi: 10.3390/biom 10070988

18. Duskaev GK, Atlanderova KN, Yausheva EV, Nurzhanov BS, Rysaev AF, Makaeva AM, Levakhin GI. Assessment of the microecological status of the rumen of cattle using the 16S Metagenomics method. IOP Conference Series: Earth and Environmental Science: IV International Scientific Conference: AGRITECH-IV-2020: Agribusiness, Environmental Engineering and Biotechnologies 18-20 November 2020, Krasnoyarsk, Russian Federation. Bristol, England: IOP Publishing; 2021;677:042010. doi: 10.1088/1755-1315/677/4/042010

19. Emami NK, Samie A, Rahmani HR, Ruiz-Feria CA. The effect of peppermint essential oil and fructooligosaccharides, as alternatives to virginiamycin, on growth performance, digestibility, gut morphology and immune response of male broilers Anim Feed Sci. Technol. 2012;175(1-2):57-64. doi: 10.1016/j.anifeedsci.2012.04.001

20. Ghosh S, Banerjee S, Sil PC. The beneficial role of curcumin on inflammation, diabetes and neurodegenerative disease: a recent update. Food Chem Toxicol. 2015;83:111-124. doi: 10.1016/j.fct.2015.05.022

21. Ghosh S, Mehla RK, Sirohi SK, Roy B. The effect of dietary garlic supplementation on body weight gain, feed intake, feed conversion efficiency, faecal score, faecal coliform count and feeding cost in crossbred dairy calves. Trop Anim Health Prod. 2010;42(5):961-968. doi: 10.1007/s11250-009-9514-5 
22. Ghosh S, Mehla RK, Sirohi SK, Tomar SK. Performance of crossbred calves with dietary supplementation of garlic extract. J Anim Physiol Anim Nutr (Berl). 2011;95(4):449-455. doi: 10.1111/j.1439-0396.2010.01071.x

23. Ghosh T, Kumar A, Sati A, Mondal BC, Singh SK, Kumar R. Effect of dietary supplementation of herbal feed additives (black cumin, garlic and turmeric) in combination with linseed oil on production performance of white leghorn laying chickens. J Entomol Zool. Stud. 2020;8(6):478-482

24. Giannenas AI, Florou-Paneri P, Papazahariadou M, Christaki E, Botsoglou NA, Spais AB. Effect of dietary supplementation with oregano essential oil on performance of broilers after experimental infection with eimeria tenella. Arch. Anim. Nutr. 2003;57(2):99106. doi: 10.1080/0003942031000107299

25. González JA, García-Barriuso M, Amich F. Ethnoveterinary medicine in the Arribes del Duero, western Spain. Vet. Res. Commun. 2011;35(5):283-310. doi: 10.1007/s11259-011-9473-y

26. Guil-Guerrero JL, Ramos L, Moreno C, Zúniga-Paredes JC, Carlosama-Yépez M, Ruales P. Antimicrobial activity of plant-food by-products: a review focusing on the tropics. Livest Sci. 2016a;189:32-49. doi: 10.1016/j.livsci.2016.04.021

27. Guil-Guerrero JL, Ramos L, Moreno C, Zúniga-Paredes JC, Carlosama-Yépez M, Ruales P. Plant-food by-products to improve farm-animal health. Anim Feed Sci Technol. 2016b;220:121-35. doi: 10.1016/j.anifeedsci.2016.07.016

28. Hashemi SR, Davoodi H. Herbal plants and their derivatives as growth and health promoters in animal nutrition Vet Res Commun. 2011;35(3):169-180. doi: 10.1007/s11259-010-9458-2

29. Hashemi SR, Davoodi H. Phytogenics as new class of feed additives in poultry industry. J Vet Anim Adv. 2010;9(17):2295-2304. doi: 10.3923/javaa.2010.2295.2304

30. Huyghebaert G, Ducatelle R, Van Immerseel F. An update on alternatives to antimicrobial growth promoters for broilers. Vet J. 2011;187(2):182-188. doi: 10.1016/j.tvj1.2010.03.003

31. Jeshari M, Riasi A, Mahdavi AH, Khorvash M, Ahmadi F. Effect of essential oils and distillation residues blends on growth performance and blood metabolites of Holstein calves weaned gradually or abruptly. Livest Sci. 2016;185:117-122. doi: 10.1016/j.livsci.2015.12.011

32. Kalayou S, Haileselassie M, Gebre-Egziabher G, Tiku'e T, Sahle S, Taddele H, Ghezu M. Invitro antimicrobial activity screening of some ethnoveterinary medicinal plants traditionally used against mastitis, wound and gastrointestinal tract complication in Tigray Region, Ethiopia. Asian Pac J Trop Biomed. 2012;2(7):512-522. doi: 10.1016/S2221-1691(12)60088-4

33. Kiczorowska B, Samolinska W, Al-Yasiry A, Kiczorowski P, Winiarska-Mieczan A. The natural feed additives as immunostimulants in monogastric animal nutrition - a review. Ann Anim Sci. 2017;17(3):605-625. doi: 10.1515/aoas-2016-0076

34. Kipre BG, Guessennd NK, Kone MW, Gbonon V, Coulibaly JK, Dosso M. Antibacterial activity of the stem bark of Tieghemella heckelii Pierre ex. A Chev against methicillin-resistant Staphylococcus aureus. BMC Complement Altern Med. 2017;17:170. doi: 10.1186/s12906-017-1681-8

35. Kumar M, Kumar V, Roy D, Kushwaha R, Vaswani S. Application of herbal feed additives in animal nutrition - a review. Int J Livest Res. 2014;4(9):1-8. doi: 10.5455/ijlr.20141205105218

36. Kumar R, Kumar BA. New claims in folk veterinary medicines from Uttar Pradesh, India. J of Ethnopharmacol. 2013;146(2):581-593. doi: 10.1016/j.jep.2013.01.030

37. Kuralkar P, Kuralkar SV. Role of herbal products in animal production - An updated review. J of Ethnopharmacology. 2021;278:114246. doi: 10.1016/j.jep.2021.114246

38. Lange CD, Pluske J, Gong J, Nyachoti C. Strategic use of feed ingredients and feed additives to stimulate gut health and development in young pigs. Livest Sci. 2010;134(1-3):124-134. doi: 10.1016/j.livsci.2010.06.117

39. Laudato M, Capasso R. Useful plants for animal therapy. OA Altern Med. 2013;1(1):1. doi: $10.13172 / 2052-7845-1-1-327$ 
Животноводство и кормопроизводство 2021 / Animal Husbandry and Fodder Production 2021;104(4) 116 ТЕОРИЯ И ПРАКТИКА КОРМЛЕНИЯ/ТНЕОRY AND PRAСТICЕ ОF FЕEDING

40. Lee KW, Everts H, Kappert HJ, Wouterse H, Frehner M, Beynen AC. Cinnamaldehyde, but not thymol, counteracts the carboxymethyl cellulose-induced growth depression in female broiler chickens. Int J Poult Sci. 2004;3(9):608-612. doi: 10.3923/ijps.2004.608.612

41. Li P, Piao X, Ru Y, Han X, Xue L, Zhang H. Effects of adding essential oil to the diet of weaned pigs on performance, nutrient utilization, immune response and intestinal health. AsianAustralasian Journal of Animal Sciences. 2012;25(11):1617-1626. doi: 10.5713/ajas.2012.12292

42. Lin J. Antibiotic growth promoters enhance animal production by targeting intestinal bile salt hydrolase and its producers. Front Microbiol. 2014;5:33. doi: 10.3389/fmicb.2014.00033

43. Luseba D, Tshisikhawe MP. Medicinal plants used in the treatment of livestock diseases in Vhembe region, Limpopo province, South Africa. J Med Plants Res. 2013;7(10):593-601. doi: 10.5897/JMPR012.1213

44. Maenner K, Vahjen W, Simon O. Studies on the effects of essential-oil-based feed additives on performance, ileal nutrient digestibility, and selected bacterial groups in the gastrointestinal tract of piglets. J Anim Sci. 2011;89(7):2106-2112. doi: 10.2527/jas.2010-2950

45. Manzanilla EG, Perez JF, Martin M, Kamel C, Baucells F, Gasa J. Effect of plant extracts and formic acid on the intestinal equilibrium of early-weaned pigs. J Anim. 2004;82(11):3210-3218. doi: 10.2527/2004.82113210x

46. Marshall BM, Levy SB. Food animals and antimicrobials: impacts on human health. Clin Microbiol. Rev. 2011;24(4):718-733. doi: 10.1128/CMR.00002-11

47. Miyasaki Y, Nichols WS, Morgan MA, Kwan JA, Van Benschoten MM, Kittell PE, Hardy WD. Screening of herbal extracts against multi-drug resistant Acinetobacter baumannii. Phytother Res. 2010;24(8):1202-1206. doi: 10.1002/ptr.3113

48. Mourao JL, Victor P, Alves A, Guedes C, de Lurdes Pinto M, Saavedra MJ, Spring P, Kocher A. Effect of mannan oligosaccharides on the performance, intestinal morphology and cecal fermentation of fattening rabbits. Anim Feed Sci Technol. 2006;126(1-2):107-120. doi: 10.1016/j.anifeedsci.2005.06.009

49. Namkung H, Li M, Gong J, Yu H, Cottrill M, De Lange CFM. Impact of feeding blends of organic acids and herbal extracts on growth performance, gut microbiota and digestive function in newly weaned pigs. Can J Anim Sci. 2004;84(4):697-704. doi: 10.4141/A04-005

50. Nanon A, Suksombat W, Yang WZ. Effects of essential oils supplementation on in vitro and in situ feed digestion in beef cattle. Anim Feed Sci Technol. 2014;196:50-59. doi: 10.1016/j.anifeedsci.2014.07.006

51. O'Bryan CA, Pendleton SJ, Crandall PG, Ricke SC. Potential of plant essential oils and their components in animal agriculture - in vitro studies on antibacterial mode of action. Front Vet Sci. 2015;2:35. doi: 10.3389/fvets.2015.00035

52. Oetting LL, Utiyama CE, Giani PA, Ruiz UD, Miyada VS. Effects of herbal extracts and antimicrobials on apparent digestibility, performance, organs morphometry and intestinal histology of weanling pigs. Braz J Anim Sci. 2006;35(4):1389-1397. doi: 10.1590/S1516-35982006000500019

53. Panda SS, Dhal NK. Plants used in ethno-veterinary medicine by native people of Nawarangpur district, Odisha, India. World J Pharm Pharm Sci. 2014;3(7):787-798.

54. Papatsiros VG, Katsoulos PD, Koutoulis KC, Karatzia M, Dedousi A, Christodoulopoulos G. Alternatives to antibiotics for farm animals. CAB Rev. 2013;8(32):1-15. doi: 10.1079/PAVSNNR20138032

55. Rahal A, Kumar A, Singh V, Yadav B, Tiwari R, Chakraborty S, Dhama K. Oxidative stress, prooxidants and antioxidants: The interplay. BioMed Res. Int. 2014;761264. doi: 10.1155/2014/761264

56. Rayzanov V, Nurzhanov B, Rysaev A, Duskaev G, Miroshnikov I. PSVIII-13 Evaluation of the effect of chlortetracycline on ruminal microbiome of ruminant against a background of plant extract. Journal of Animal Science. 2020;98(S4):258-259. doi: 10.1093/jas/skaa278.467

57. Reddy MK, Gupta SK, Jacob MR, Khan SI, Ferreira D. Antioxidant, antimalarial and antimicrobial activities of tannin-rich fractions, ellagitannins and phenolic acids from Punica granatum L. Planta. Med. 2007;73(5):461-467. doi: 10.1055/s-2007-967167 
58. Ryazanov VA, Duskaev GK, Rakhmatullin ShG, Miroshnikov IS, Miroshnikova KP, Inchagova KS. Application of new technologies to assess the effectiveness of feed materials for ruminants. IOP Conference Series: Earth and Environmental Science: International Conference on World Technological Trends in Agribusiness 4-5 July 2020, Omsk City, Western Siberia, Russian Federation. Bristol, England: IOP Publishing; 2021;624:012049. doi: 10.1088/1755-1315/624/1/012049

59. Saeed M, Naveed M, Leskovec J, Ali Kamboh A, Kakar I, Ullah K, Ahmad F, Sharif M, Javaid A, Rauf M, Abd El-Hack ME, Abdel-Latif MA, Chao S. Using guduchi (Tinospora cordifolia) as an eco-friendly feed supplement in human and poultry nutrition. Poultry Sci. 2020;99(2):801-811. doi: 10.1016/j.psj.2019.10.051

60. Sarica S, Ciftci A, Demir E, Kilinc K, Yildirim Y. Use of an antibiotic growth promoter and two herbal natural feed additives with and without exogenous enzymes in wheat based broiler diets. S Afr J Anim Sci. 2005;35(1):61-72. doi: 10.4314/sajas.v35i1.4050

61. Seal BS, Lillehoj HS, Donovan DM, Gay CG. Alternatives to antibiotics: a symposium on the challenges and solutions for animal production. Anim Health Res. Rev. 2013;14(1):78-87. doi: $10.1017 /$ S1466252313000030

62. Sethiya NK. Review on natural growth promoters available for improving gut health of poultry: an alternative to antibiotic growth promoters. Asian J. Poultry Sci. 2016;10(1):1-29. doi: 10.3923/ajpsaj.2016.1.29

63. Singh J, Gaikwad DS. Phytogenic feed additives in animal nutrition. In: Singh, A. Yadav, editos. Natural bioactive products in sustainable agriculture. Singapore: Springer. 2020:273-289. doi: 10.1007/978-981-15-3024-1_13

64. Singh PK, Kumar A, Tiwari DP. Effects of dietary supplementation of black cumin, garlic and turmeric on the production performance and egg quality of White Leghorn hens. Anim Nutr Feed Technol. 2019;19(3):361-370. doi: 10.5958/0974-181X.2019.00034.9

65. Stanton TB. A call for antibiotic alternatives research. Trends Microbiol. 2013;21(3):111-113. doi: $10.1016 /$ j.tim.2012.11.002

66. Stavri M, Piddock LJ, Gibbons S. Bacterial efflux pump inhibitors from natural sources. J Antimicrob Chemother. 2007;59(6):1247-1260. doi: 10.1093/jac/dk1460

67. Stevanović ZD, Bošnjak-Neumüller J, Pajić-Lijaković I, Raj J, Vasiljević M. Essential oils as feed additives - Future perspectives. Molecules. 2018;23(7):1717. doi: 10.3390/molecules23071717

68. Taga I, Lan CQ, Altosaar I. Plant essential oils and mastitis disease: their potential inhibitory effects on pro-inflammatory cytokine production in response to bacteria related inflammation. Nat Prod Commun. 2012;7(5):675-682.

69. Tapki I, Ozalpaydin HB, Tapki N, Aslan M, Selvi MH. Effects of oregano essential oil on reduction of weaning age and increasing economic efficiency in Holstein Friesian calves. Pakistan Journal of Zoology. 2020;52(2):745-752 doi: 10.17582/journal.pjz/20180606130639

70. Upadhyaya I, Upadhyay A, Kollanoor-Johny A, Mooyottu S, Baskaran SA, Yin HB, Schreiber DT, Khan MI, Darre MJ, Curtis PA, Venkitanarayanan K. In-feed supplementation of trans-cinnamaldehyde reduces layer-chicken egg-borne transmission of Salmonella enterica serovar enteritidis. Appl Environ Microbiol. 2015;81(9):2985-2994. doi: 10.1128/AEM.03809-14

71. Viegi L, Pieroni A, Guarrera PM, Vangelisti RA. A review of plants used in folk veterinary medicine in Italy as basis for a databank J Ethnopharmacol. 2003;89(2 -3):221-244. doi: $10.1016 /$ j.jep.2003.08.003

72. Windisch W, Schedle K, Plitzer C, Kroismayr A.Use of phytogenic products as feed additives for swine and poultry. J Anim Sci. 2008;6(S14):E140-E148. doi: 10.2527/jas.2007-0459

73. Yan L, Meng QW, Kim IH. The effect of an herb extract mixture on growth performance, nutrient digestibility, blood characteristics and fecal noxious gas content in growing pigs. Livest Sci. 2011;141(2-3):143-147. doi: 10.1016/j.livsci.2011.05.011 
Животноводство и кормопроизводство 2021 / Animal Husbandry and Fodder Production 2021;104(4) 118 ТЕОРИЯ И ПРАКТИКА КОРМЛЕНИЯ/ТНЕОRY AND PRAСТICЕ ОF FЕEDING

74. Yang Y, Iji P, Choct M. Dietary modulation of gut microflora in broiler chickens: a review of the role of six kinds of alternatives to in-feed antibiotics. World Poultry Sci J. 2009;65(1):97-114. doi: $10.1017 /$ S0043933909000087

75. Zeng Z, Zhang S, Wang H, Piao X. Essential oil and aromatic plants as feed additives in nonruminant nutrition: a review. J Anim Sci Biotechnol. 2015;6(7):1-10. doi: 10.1186/s40104-015-0004-5

\section{References}

1. Duskaev GK, Levakhin GI, Korolyov VL, Sirazetdinov FKh. Use of probiotics and plant extracts to improve the productivity of ruminants (review). Animal Husbandry and Fodder Production. 2019;102(1):136-148. doi: 10.33284/2658-3135-102-1-136

2. Atlanderova KN, Duskaev GK, Makaeva AM, Muslyumova DM, Kondrashova KS. Cattle rumen microbiome after Quercus cortex extract. Animal Husbandry and Fodder Production. 2019;102(4):186-197. doi: 10.33284 / 2658-3135-102-4-186

3. Bagno OA, Prokhorov ON, Shevchenko SA, Shevchenko AI, Dyadichkina TV. Use of phytobioticts in farm animal feeding (review). [Agricultural Biology]. 2018;53(4):687-697. (In Russ)]. doi: 10.15389/agrobiology.2018.4.687eng

4. Abreu AC, McBain AJ, Simoes M. Plants as sources of new antimicrobials and resistancemodifying agents. Nat Prod Rep. 2012(9);29:1007-1021. doi: 10.1039/c2np20035j

5. Adebiyi OA, Ajayi OS, Adejumo IO, Osungade TO. Performance, microbial load and gut morphology of weaned pigs fed diets supplemented with turmeric, ginger and garlic extract. Trop Anim Prod. Investig. 2014;17(1):25-31.

6. Al-Kassie GAM. The role of peppermint (Mentha piperita) on performance in broiler diets. Agric Biol J N Am. 2010;1(5):1009-1013.

7. Atlanderova KN, Makaeva AM, Kurilkina MYa, Kholodilina TN, Duskaev GK. Technology for increasing the bioavailability of feed using quorum sensing inhibitors. IOP Conference Series: Earth and Environmental Science: International Conference on World Technological Trends in Agribusiness 4-5 July 2020, Omsk City, Western Siberia, Russian Federation. Bristol, England: IOP Publishing; 2021;624:012159. doi: 10.1088/1755-1315/624/1/012159

8. Atlanderova KN, Makaeva AM, Sizova EA, Duskaev GK. Stimulation of ruminal digestion of young cattle with oak bark extract (Quercus cortex). IOP Conference Series: Earth and Environmental Science: Conference on Innovations in Agricultural and Rural development; 2019 Apr 18-19; Kurgan, Russian Federation. Bristol, England: IOP Publishing; 2019a;341:012059. doi: 10.1088/1755-1315/341/1/012059

9. Atlanderova KN, Makaeva AM, Sizova EA. Comparative evaluation of the effect of the Quercus cortex extract and biologically active substances of plant origin on health and scar digestion. IOP Conference Series: Earth and Environmental Science: Conference on Innovations in Agricultural and Rural development; 2019 Apr 18-19; Kurgan, Russian Federation. Bristol, England: IOP Publishing; 2019b;341:012189. doi: 10.1088/1755-1315/341/1/012189

10. Borchardt RA, Rolston KV. Antibiotic shortages: effective alternatives in the face of a growing problem. Jaapa. 2013;26(2):13-18. doi: 10.1097/01720610-201302000-00004

11. Castanon JI. History of the use of antibiotic as growth promoters in European poultry feeds. Poult. Sci. 2007;86(11):2466-2471. doi: 10.3382/ps.2007-00249

12. Cheng G, Hao H, Xie S, Wang X, Dai M, Huang L, Yuan Z. Antibiotic alternatives: the substitution of antibiotics in animal husbandry? Front Microbiol. 2014;5:69-83. doi: $10.3389 /$ fmicb.2014.00217

13. Cooper MA, Shlaes D. Fix the antibiotics pipeline. Nature. 2011;472:32. doi: $10.1038 / 472032 \mathrm{a}$

14. Costa LB, Panhoza Tse ML, Miyada VS. Herbal extracts as alternatives to antimicrobial growth for weanling pigs. Braz J Anim Sci. 2007;36(3):589-595 doi: 10.1590/S1516-35982007000300011 
15. Cross DE, Mcdevitt RM, Hillman K, Acamovic T. The effect of herbs and their associated essential oils on performance, dietary digestibility and gut microflora in chickens from 7 to 28 days of age. Br Poult Sci. 2007;48(4):496-506. doi: 10.1080/00071660701463221

16. Dhama K, Tiwari R, Chakraborty S, Saminathan M, Kumar A, Karthik K, Yaqoob M, Amarpal W, Singh SV, Rahal A. Evidence based antibacterial potentials of medicinal plants and herbs countering bacterial pathogens especially in the era of emerging drug resistance: an integrated update. Int $\mathrm{J}$ pharmacology. 2014;10(1):1-43. doi: 10.3923/ijp.2014.1.43

17. Diniz do Nascimento L, Moraes A, Costa K, Pereira JM, Galúcio, Taube PS, Costa C, Neves Cruz J, de Aguiar Andrade EH, Faria L. Bioactive natural compounds and antioxidant activity of essential oils from spice plants: new findings and potential applications Biomolecules. 2020;10(7):988. doi: 10.3390/biom10070988

18. Duskaev GK, Atlanderova KN, Yausheva EV, Nurzhanov BS, Rysaev AF, Makaeva AM, Levakhin GI. Assessment of the microecological status of the rumen of cattle using the 16S Metagenomics method. IOP Conference Series: Earth and Environmental Science: IV International Scientific Conference: AGRITECH-IV-2020: Agribusiness, Environmental Engineering and Biotechnologies 18-20 November 2020, Krasnoyarsk, Russian Federation. Bristol, England: IOP Publishing; 2021;677:042010. doi: 10.1088/1755-1315/677/4/042010

19. Emami NK, Samie A, Rahmani HR, Ruiz-Feria CA. The effect of peppermint essential oil and fructooligosaccharides, as alternatives to virginiamycin, on growth performance, digestibility, gut morphology and immune response of male broilers Anim Feed Sci. Technol. 2012;175(1-2):57-64. doi: 10.1016/j.anifeedsci.2012.04.001

20. Ghosh S, Banerjee S, Sil PC. The beneficial role of curcumin on inflammation, diabetes and neurodegenerative disease: a recent update. Food Chem Toxicol. 2015;83:111-124. doi: 10.1016/j.fct.2015.05.022

21. Ghosh S, Mehla RK, Sirohi SK, Roy B. The effect of dietary garlic supplementation on body weight gain, feed intake, feed conversion efficiency, faecal score, faecal coliform count and feeding cost in crossbred dairy calves. Trop Anim Health Prod. 2010;42(5):961-968. doi: 10.1007/s11250-009-9514-5

22. Ghosh S, Mehla RK, Sirohi SK, Tomar SK. Performance of crossbred calves with dietary supplementation of garlic extract. J Anim Physiol Anim Nutr (Berl). 2011;95(4):449-455. doi: 10.1111/j.1439-0396.2010.01071.x

23. Ghosh T, Kumar A, Sati A, Mondal BC, Singh SK, Kumar R. Effect of dietary supplementation of herbal feed additives (black cumin, garlic and turmeric) in combination with linseed oil on production performance of white leghorn laying chickens. J Entomol Zool. Stud. 2020;8(6):478-482

24. Giannenas AI, Florou-Paneri P, Papazahariadou M, Christaki E, Botsoglou NA, Spais AB. Effect of dietary supplementation with oregano essential oil on performance of broilers after experimental infection with eimeria tenella. Arch. Anim. Nutr. 2003;57(2):99106. doi: 10.1080/0003942031000107299

25. González JA, García-Barriuso M, Amich F. Ethnoveterinary medicine in the Arribes del Duero, western Spain. Vet. Res. Commun. 2011;35(5):283-310. doi: 10.1007/s11259-011-9473-y

26. Guil-Guerrero JL, Ramos L, Moreno C, Zúniga-Paredes JC, Carlosama-Yépez M, Ruales P. Antimicrobial activity of plant-food by-products: a review focusing on the tropics. Livest Sci. 2016a;189:32-49. doi: 10.1016/j.livsci.2016.04.021

27. Guil-Guerrero JL, Ramos L, Moreno C, Zúniga-Paredes JC, Carlosama-Yépez M, Ruales P. Plant-food by-products to improve farm-animal health. Anim Feed Sci Technol. 2016b;220:121-35. doi: 10.1016/j.anifeedsci.2016.07.016

28. Hashemi SR, Davoodi H. Herbal plants and their derivatives as growth and health promoters in animal nutrition Vet Res Commun. 2011;35(3):169-180. doi: 10.1007/s11259-010-9458-2

29. Hashemi SR, Davoodi H. Phytogenics as new class of feed additives in poultry industry. J Vet Anim Adv. 2010;9(17):2295-2304. doi: 10.3923/javaa.2010.2295.2304

30. Huyghebaert G, Ducatelle R, Van Immerseel F. An update on alternatives to antimicrobial growth promoters for broilers. Vet J. 2011;187(2):182-188. doi: 10.1016/j.tvj1.2010.03.003 
Животноводство и кормопроизводство 2021 / Animal Husbandry and Fodder Production 2021;104(4) $\underline{120}$ ТЕОРИЯ И ПРАКТИКА КОРМЛЕНИЯ/ТНЕОRY AND РRAСТІСЕ ОF FЕЕDING

31. Jeshari M, Riasi A, Mahdavi AH, Khorvash M, Ahmadi F. Effect of essential oils and distillation residues blends on growth performance and blood metabolites of Holstein calves weaned gradually or abruptly. Livest Sci. 2016;185:117-122. doi: 10.1016/j.livsci.2015.12.011

32. Kalayou S, Haileselassie M, Gebre-Egziabher G, Tiku'e T, Sahle S, Taddele H, Ghezu M. Invitro antimicrobial activity screening of some ethnoveterinary medicinal plants traditionally used against mastitis, wound and gastrointestinal tract complication in Tigray Region, Ethiopia. Asian Pac J Trop Biomed. 2012;2(7):512-522. doi: 10.1016/S2221-1691(12)60088-4

33. Kiczorowska B, Samolinska W, Al-Yasiry A, Kiczorowski P, Winiarska-Mieczan A. The natural feed additives as immunostimulants in monogastric animal nutrition - a review. Ann Anim Sci. 2017;17(3):605-625. doi: 10.1515/aoas-2016-0076

34. Kipre BG, Guessennd NK, Kone MW, Gbonon V, Coulibaly JK, Dosso M. Antibacterial activity of the stem bark of Tieghemella heckelii Pierre ex. A Chev against methicillin-resistant Staphylococcus aureus. BMC Complement Altern Med. 2017;17:170. doi: 10.1186/s12906-017-1681-8

35. Kumar M, Kumar V, Roy D, Kushwaha R, Vaswani S. Application of herbal feed additives in animal nutrition - a review. Int J Livest Res. 2014;4(9):1-8. doi: 10.5455/ijlr.20141205105218

36. Kumar R, Kumar BA. New claims in folk veterinary medicines from Uttar Pradesh, India. J of Ethnopharmacol. 2013;146(2):581-593. doi: 10.1016/j.jep.2013.01.030

37. Kuralkar P, Kuralkar SV. Role of herbal products in animal production - An updated review. J of Ethnopharmacology. 2021;278:114246. doi: 10.1016/j.jep.2021.114246

38. Lange CD, Pluske J, Gong J, Nyachoti C. Strategic use of feed ingredients and feed additives to stimulate gut health and development in young pigs. Livest Sci. 2010;134(1-3):124-134. doi: 10.1016/j.livsci.2010.06.117

39. Laudato M, Capasso R. Useful plants for animal therapy. OA Altern Med. 2013;1(1):1. doi: $10.13172 / 2052-7845-1-1-327$

40. Lee KW, Everts H, Kappert HJ, Wouterse H, Frehner M, Beynen AC. Cinnamaldehyde, but not thymol, counteracts the carboxymethyl cellulose-induced growth depression in female broiler chickens. Int J Poult Sci. 2004;3(9):608-612. doi: 10.3923/ijps.2004.608.612

41. Li P, Piao X, Ru Y, Han X, Xue L, Zhang H. Effects of adding essential oil to the diet of weaned pigs on performance, nutrient utilization, immune response and intestinal health. AsianAustralasian Journal of Animal Sciences. 2012;25(11):1617-1626. doi: 10.5713/ajas.2012.12292

42. Lin J. Antibiotic growth promoters enhance animal production by targeting intestinal bile salt hydrolase and its producers. Front Microbiol. 2014;5:33. doi: 10.3389/fmicb.2014.00033

43. Luseba D, Tshisikhawe MP. Medicinal plants used in the treatment of livestock diseases in Vhembe region, Limpopo province, South Africa. J Med Plants Res. 2013;7(10):593-601. doi: 10.5897/JMPR012.1213

44. Maenner K, Vahjen W, Simon O. Studies on the effects of essential-oil-based feed additives on performance, ileal nutrient digestibility, and selected bacterial groups in the gastrointestinal tract of piglets. J Anim Sci. 2011;89(7):2106-2112. doi: 10.2527/jas.2010-2950

45. Manzanilla EG, Perez JF, Martin M, Kamel C, Baucells F, Gasa J. Effect of plant extracts and formic acid on the intestinal equilibrium of early-weaned pigs. J Anim. 2004;82(11):3210-3218. doi: 10.2527/2004.82113210x

46. Marshall BM, Levy SB. Food animals and antimicrobials: impacts on human health. Clin Microbiol. Rev. 2011;24(4):718-733. doi: 10.1128/CMR.00002-11

47. Miyasaki Y, Nichols WS, Morgan MA, Kwan JA, Van Benschoten MM, Kittell PE, Hardy WD. Screening of herbal extracts against multi-drug resistant Acinetobacter baumannii. Phytother Res. 2010;24(8):1202-1206. doi: 10.1002/ptr.3113

48. Mourao JL, Victor P, Alves A, Guedes C, de Lurdes Pinto M, Saavedra MJ, Spring P, Kocher A. Effect of mannan oligosaccharides on the performance, intestinal morphology and cecal fermentation of fattening rabbits. Anim Feed Sci Technol. 2006;126(1-2):107-120. doi: 10.1016/j.anifeedsci.2005.06.009 
49. Namkung H, Li M, Gong J, Yu H, Cottrill M, De Lange CFM. Impact of feeding blends of organic acids and herbal extracts on growth performance, gut microbiota and digestive function in newly weaned pigs. Can J Anim Sci. 2004;84(4):697-704. doi: 10.4141/A04-005

50. Nanon A, Suksombat W, Yang WZ. Effects of essential oils supplementation on in vitro and in situ feed digestion in beef cattle. Anim Feed Sci Technol. 2014;196:50-59. doi: 10.1016/j.anifeedsci.2014.07.006

51. O'Bryan CA, Pendleton SJ, Crandall PG, Ricke SC. Potential of plant essential oils and their components in animal agriculture - in vitro studies on antibacterial mode of action. Front Vet Sci. 2015;2:35. doi: 10.3389/fvets.2015.00035

52. Oetting LL, Utiyama CE, Giani PA, Ruiz UD, Miyada VS. Effects of herbal extracts and antimicrobials on apparent digestibility, performance, organs morphometry and intestinal histology of weanling pigs. Braz J Anim Sci. 2006;35(4):1389-1397. doi: 10.1590/S1516-35982006000500019

53. Panda SS, Dhal NK. Plants used in ethno-veterinary medicine by native people of Nawarangpur district, Odisha, India. World J Pharm Pharm Sci. 2014;3(7):787-798.

54. Papatsiros VG, Katsoulos PD, Koutoulis KC, Karatzia M, Dedousi A, Christodoulopoulos G. Alternatives to antibiotics for farm animals. CAB Rev. 2013;8(32):1-15. doi: 10.1079/PAVSNNR20138032

55. Rahal A, Kumar A, Singh V, Yadav B, Tiwari R, Chakraborty S, Dhama K. Oxidative stress, prooxidants and antioxidants: The interplay. BioMed Res. Int. 2014;761264. doi: 10.1155/2014/761264

56. Rayzanov V, Nurzhanov B, Rysaev A, Duskaev G, Miroshnikov I. PSVIII-13 Evaluation of the effect of chlortetracycline on ruminal microbiome of ruminant against a background of plant extract. Journal of Animal Science. 2020;98(S4):258-259. doi: 10.1093/jas/skaa278.467

57. Reddy MK, Gupta SK, Jacob MR, Khan SI, Ferreira D. Antioxidant, antimalarial and antimicrobial activities of tannin-rich fractions, ellagitannins and phenolic acids from Punica granatum L. Planta. Med. 2007;73(5):461-467. doi: 10.1055/s-2007-967167

58. Ryazanov VA, Duskaev GK, Rakhmatullin ShG, Miroshnikov IS, Miroshnikova KP, Inchagova KS. Application of new technologies to assess the effectiveness of feed materials for ruminants. IOP Conference Series: Earth and Environmental Science: International Conference on World Technological Trends in Agribusiness 4-5 July 2020, Omsk City, Western Siberia, Russian Federation. Bristol, England: IOP Publishing; 2021;624:012049. doi: 10.1088/1755-1315/624/1/012049

59. Saeed M, Naveed M, Leskovec J, Ali Kamboh A, Kakar I, Ullah K, Ahmad F, Sharif M, Javaid A, Rauf M, Abd El-Hack ME, Abdel-Latif MA, Chao S. Using guduchi (Tinospora cordifolia) as an eco-friendly feed supplement in human and poultry nutrition. Poultry Sci. 2020;99(2):801-811. doi: 10.1016/j.psj.2019.10.051

60. Sarica S, Ciftci A, Demir E, Kilinc K, Yildirim Y. Use of an antibiotic growth promoter and two herbal natural feed additives with and without exogenous enzymes in wheat based broiler diets. S Afr J Anim Sci. 2005;35(1):61-72. doi: 10.4314/sajas.v35i1.4050

61. Seal BS, Lillehoj HS, Donovan DM, Gay CG. Alternatives to antibiotics: a symposium on the challenges and solutions for animal production. Anim Health Res. Rev. 2013;14(1):78-87. doi: $10.1017 / \mathrm{S} 1466252313000030$

62. Sethiya NK. Review on natural growth promoters available for improving gut health of poultry: an alternative to antibiotic growth promoters. Asian J. Poultry Sci. 2016;10(1):1-29. doi: 10.3923/ajpsaj.2016.1.29

63. Singh J, Gaikwad DS. Phytogenic feed additives in animal nutrition. In: Singh, A. Yadav, editos. Natural bioactive products in sustainable agriculture. Singapore: Springer. 2020:273-289. doi: 10.1007/978-981-15-3024-1_13

64. Singh PK, Kumar A, Tiwari DP. Effects of dietary supplementation of black cumin, garlic and turmeric on the production performance and egg quality of White Leghorn hens. Anim Nutr Feed Technol. 2019;19(3):361-370. doi: 10.5958/0974-181X.2019.00034.9

65. Stanton TB. A call for antibiotic alternatives research. Trends Microbiol. 2013;21(3):111-113. doi: $10.1016 /$ j.tim.2012.11.002 
Животноводство и кормопроизводство 2021 / Animal Husbandry and Fodder Production 2021;104(4) 122 ТЕОРИЯ И ПРАКТИКА КОРМЛЕНИЯ/ТНЕОRY AND PRAСТICЕ ОF FЕEDING

66. Stavri M, Piddock LJ, Gibbons S. Bacterial efflux pump inhibitors from natural sources. J Antimicrob Chemother. 2007;59(6):1247-1260. doi: 10.1093/jac/dk1460

67. Stevanović ZD, Bošnjak-Neumüller J, Pajić-Lijaković I, Raj J, Vasiljević M. Essential oils as feed additives - Future perspectives. Molecules. 2018;23(7):1717. doi: 10.3390/molecules23071717

68. Taga I, Lan CQ, Altosaar I. Plant essential oils and mastitis disease: their potential inhibitory effects on pro-inflammatory cytokine production in response to bacteria related inflammation. Nat Prod Commun. 2012;7(5):675-682.

69. Tapki I, Ozalpaydin HB, Tapki N, Aslan M, Selvi MH. Effects of oregano essential oil on reduction of weaning age and increasing economic efficiency in Holstein Friesian calves. Pakistan Journal of Zoology. 2020;52(2):745-752 doi: 10.17582/journal.pjz/20180606130639

70. Upadhyaya I, Upadhyay A, Kollanoor-Johny A, Mooyottu S, Baskaran SA, Yin HB, Schreiber DT, Khan MI, Darre MJ, Curtis PA, Venkitanarayanan K. In-feed supplementation of trans-cinnamaldehyde reduces layer-chicken egg-borne transmission of Salmonella enterica serovar enteritidis. Appl Environ Microbiol. 2015;81(9):2985-2994. doi: 10.1128/AEM.03809-14

71. Viegi L, Pieroni A, Guarrera PM, Vangelisti RA. A review of plants used in folk veterinary medicine in Italy as basis for a databank J Ethnopharmacol. 2003;89(2-3):221-244. doi: $10.1016 / \mathrm{j}$.jep.2003.08.003

72. Windisch W, Schedle K, Plitzer C, Kroismayr A.Use of phytogenic products as feed additives for swine and poultry. J Anim Sci. 2008;6(S14):E140-E148. doi: 10.2527/jas.2007-0459

73. Yan L, Meng QW, Kim IH. The effect of an herb extract mixture on growth performance, nutrient digestibility, blood characteristics and fecal noxious gas content in growing pigs. Livest Sci. 2011;141(2-3):143-147. doi: 10.1016/j.livsci.2011.05.011

74. Yang Y, Iji P, Choct M. Dietary modulation of gut microflora in broiler chickens: a review of the role of six kinds of alternatives to in-feed antibiotics. World Poultry Sci J. 2009;65(1):97-114. doi: $10.1017 /$ S0043933909000087

75. Zeng Z, Zhang S, Wang H, Piao X. Essential oil and aromatic plants as feed additives in nonruminant nutrition: a review. J Anim Sci Biotechnol. 2015;6(7):1-10. doi: 10.1186/s40104-015-0004-5

\section{Информация об авторах:}

Виталий Александрович Рязанов, кандидат сельскохозяйственных наук, научный сотрудник отдела кормления сельскохозяйственных животных и технологии кормов им. С.Г. Леушина, Федеральный научный центр биологических систем и агротехнологий Российской академии наук, 460000, г. Оренбург, ул. 9 Января, 29, тел.: 8-922-807-71-00.

Марина Яковлевна Курилкина, кандидат биологических наук, старший научный сотрудник Испытательного центра, Федеральный научный центр биологических систем и агротехнологий Российской академии наук, 460000, г. Оренбург, ул. 9 Января, 29, тел.: 8(3532)30-81-77.

Галимжан Калиханович Дускаев, доктор биологических наук, ведущий научный сотрудник отдела кормления сельскохозяйственных животных и технологии кормов им. С.Г. Леушина, Федеральный научный центр биологических систем и агротехнологий Российской академии наук; 460000, г. Оренбург, ул. 9 Января, 29, тел.: +7 (3532)30-81-79.

Вячеслав Михайлович Габидулин, доктор сельскохозяйственных наук, ведущий научный сотрудник отдела разведения мясного скота, Федеральный научный центр биологических систем и агротехнологий Российской академии наук, 460000, г. Оренбург, ул. 9 Января, 29, тел.: 8(3532)3081-74.

\section{Information about the authors:}

Vitaly A Ryazanov, Cand. Sci. (Agriculture), Researcher of Farm Animal Feeding and Feed Technology Department named after Leushin SG, Federal Research Centre of Biological Systems and Agrotechnologies of the Russian Academy of Sciences, 460000, Orenburg, 29, 9 Yanvarya St., tel.: 8(3532)30-81-79. 
Marina Ya Kurilkina, Cand. Sci. (Biology), Senior Researcher at the Testing Centre of the Common Use Centre, Federal Research Centre of Biological Systems and Agrotechnologies of the Russian Academy of Sciences, 460000, Orenburg, Russia, 29, 9 Yanvarya St., tel.: 8(3532)30-81-77.

Galimzhan K Duskaev, Dr. Sci. (Biology), Leading Researcher, Farm Animal Feeding and Feed Technology Department named after Leushin SG, Federal Research Centre of Biological Systems and Agrotechnologies of the Russian Academy of Sciences, 460000, Orenburg, Russia, 29, 9 Yanvarya St., tel.: 8(3532)30-81-79, e-mail: gduskaev@mail.ru.

Vyacheslav M Gabidulin, Dr. Sci. (Agriculture), Leading Researcher, Beef Cattle Breeding Department, Federal Research Centre of Biological Systems and Agrotechnologies of the Russian Academy of Sciences, 460000, Orenburg, Russia, 29, 9 Yanvarya St., tel.: 8(3532)30-81-74.

Статья поступила в редакцию 17.11.2021; одобрена после рецензирования 26.11.2021; принята к публикации 13.12.2021.

The article was submitted 17.11.2021; approved after reviewing 26.11.2021; accepted for publication 13.12.2021. 